\title{
Hall Effect on Two-Phase Laminar Boundary Layer flow of Dusty Liquid due to Stretching of an Elastic Flat Sheet
}

\author{
B Mahanthesh*
}

\begin{abstract}
The present investigation is concerned with the effect of Hall current on boundary layer two-phase flow of an electrically conducting dusty fluid over a permeable stretching sheet in the presence of a strong magnetic field. The boundary layer approximation is employed for mathematical modeling. The governing partial differential equations are reduced to a set of ordinary differential equations using suitable similarity transformations. Subsequent equations are solved numerically by using Runge-Kutta-Fehlberg fourth-fifth order method. A comprehensive parametric study is conducted to reveal the tendency of solutions. It is found that the mass concentration of dust particles can be used as a control parameter to control the friction factor at the sheet. The influence of suction and injection are opposite on the momentum boundary layer growth.
\end{abstract}

Keywords: Boundary layer flow, dusty fluid, numerical solution, Hall effect, suction/blowing, stretching sheet.

Mathematics Subject Classification (2010): 76T15, 80A20

\section{Introduction}

The magnetic field effect on the laminar flow of an electrically conducting fluid plays an important role in the process of purification of molten metals from non-metallic inclusions. Many works have been reported on flow and heat transfer of an electrically conducting fluid

*Department of Mathematics, Christ University, India, 560029; mahanthesh.b@christuniversity.in 
over a stretched surface in the presence of a magnetic field. In an ionized gas where the density is low, the magnetic field is very strong; the conductivity will be a tensor. The conductivity normal to the magnetic field is reduced due to the free spiraling of electrons and ions about the magnetic lines of force before suffering collisions and a current is induced in a direction normal to both the electric and magnetic fields. This phenomenon is called the Hall effect. When the medium is rarefied or if the strong magnetic field is present, the conductivity of the fluid is anisotropic and the effect of Hall current cannot be ignored. The study of MHD flows with hall current has important applications in the problem of Hall accelerators as well as flight magnetohydrodynamics. The current trend for the application of magnetohydrodynamics is toward a strong magnetic field (so that the effect of electromagnetic force noticeable) and towards a low density of the gas (such as in space flight and in nuclear fusion research). Under this condition Hall effect becomes important. With this understanding Sato[1], Yamanishi[2] have studied the MHD flow of a viscous fluid through a channel by considering the Hall effect. Then after, Pop[3] studied the effects of Hall current on hydrodynamic flow near a porous plate. Hydrodynamic free convection and mass transfer of an electrically conducting viscous fluid past an infinite vertical porous plate have been reported by Singh and Gorla. [4] Abd El Aziz[5] investigated the effect of hall currents on the flow and heat transfer of an electrically conducting fluid over an unsteady stretching sheet in the presence of a strong magnetic field. Ali et al. [6] studied the influence of Hall current on MHD mixed convection boundary layer flow over a stretched vertical flat plate. Hall current and Ohmic heating effects on the mixed convection boundary-layer flow of a micropolar fluid from a rotating cone with power-law variation in surface temperature have investigated by Abd El Aziz et al. [7] Recently, Gireesha et al. [8] presented a perturbation analysis of radiating viscoelastic fluid flow and heat transfer in a non-uniform channel with Hall current and chemical reaction.

Mention can be made that these studies concern boundary layer behavior of only ordinary viscous fluids over horizontal/vertical stretching sheet. However, by nature, air, water, and many other fluids contain impurities like dust particles and foreign bodies. This binary mixture of viscous fluid and solid particles is called dusty fluid. In fact, the problem of two-phase flow in which solid particles are distributed in clean fluid has many practical applications such as purification of crude oil, petroleum industry, nuclear processing, combustion, use of dust in gas cooling systems, centrifugal separation of matter from a fluid, electrostatic precipitation and in polymer technology. In view of these applications, Saffman[9] proposed a mathematical model for dusty fluid flow which was also considered by various authors. Following Saffman model, Liu[10] investigated the flow induced by an 
oscillating infinite flat plate in a dusty gas. Chakraborti[11] analyzed the boundary layer flow of a dusty gas. Later, Datta and Mishra[12] have investigated dusty fluid in boundary layer flow over the semiinfinite flat plate. Then after, hydromagnetic flow and heat transfer of a dusty fluid over stretching sheet with suction effect has been investigated by Vajravelu and Nayef.[13] Further, Xie et al.[14] have extended the work of [13] and studied on the hydromagnetic stability of a particle-laden flow in growing flat plate boundary layer. Palani and Ganesan[15] have studied heat transfer effects on dusty gas flow past a semi-infinite inclined plate. They found that the clean fluid velocity and temperature profile is higher than that of dusty fluid. More recently, Gireesha et al.[16] studied the Maxwell fluid under the influence of non-linear thermal radiation and nonuniform heat source/sink. Sandeep et al. [17] have numerically analyzed the unsteady radiative flow and heat transfer characteristics of a dusty nanofluid over an exponentially permeable stretching surface in presence of volume fraction of dust and nano particles. Siddiqa et al. [18] numerically reported the natural convection flow of a twophase dusty nanofluid along a vertical wavy frustum of a cone. The mixed convection dusty fluid flow past a vertical wedge due to a small fluctuation in free stream and surface temperature for the unsteady flow was examined by Hossain et al. [19] Very recently, Mustafa[20] reported the analytical solution for two-phase dusty fluid flow and heat model over deforming isothermal surfaces.

A close observation of the literature reveals that there are some missing links, i.e., there are studies which do not consider the effect of Hall current on the hydrodynamic two-phase boundary layer of an electrically conducting dusty viscous fluid over a permeable stretching surface in the presence of suction/blowing. The present paper attempts to fill this lacuna. To the best of authors' knowledge, so far no one has considered this investigation. In this article, we employ an extensively validated, highly efficient numerical method called, RungeKutta-Fehlberg fourth-fifth order method to study this problem. The influence of various interesting parameters on the flow characteristics is presented and discussed.

\section{Mathematical Formulation}

Consider the steady flow of a viscous, incompressible and electrically conducting dusty fluid past a permeable stretching sheet coinciding with the plane $y=0$. The fluid may be a water or ethylene glycol embedded with micro sized electrically conducting dust particles. The dusty liquid is occupied above the sheet yi0. A schematic representation of the physical model and coordinates system is depicted in Fig. 1. 


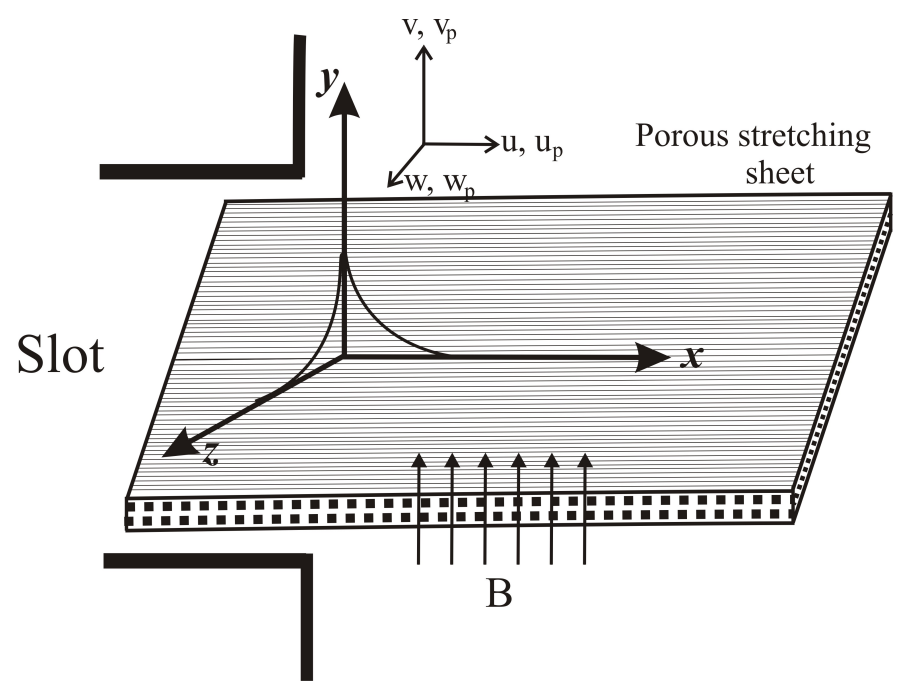

Figure 1: A schematic representation of the physical model and coordinates system.

The positive $\mathrm{x}$-coordinate is measured along the stretching sheet in the direction of motion and the positive y-coordinate is measured normal to the sheet in the outward direction toward the fluid. The leading edge of the stretching sheet is taken as coincident with z-axis. An external strong magnetic field is applied in the positive y-direction. The effect of Hall current gives rise to a force in the z-direction, which induces a cross flow in that direction and hence the flow becomes three-dimensional. To simplify the problem, we assume that there is no variation of flow quantities in z-direction. This assumption is considered to be valid if the surface be of infinite extent in the $\mathrm{z}$ direction. The generalized Ohms law including Hall currents is given in the form[6];

$\vec{J}+\frac{\omega_{e} \tau_{e}}{B_{0}}(\vec{J} \times \vec{B})=\sigma\left(\vec{E}+\vec{V} \times \vec{B}+\frac{1}{e n_{e}} \nabla p_{e}\right)$

where $\vec{J}=\left(J_{x}, J_{y}, J_{z}\right)$ is the current density vector, $\vec{V}$ is the velocity vector, $\vec{E}$ is the electric field vector, $\vec{B}=\left(0, B_{0}, 0\right)$ is the magnetic induction vector, $\sigma$ is the electrical conductivity, $\tau_{e}$ is the electron collision time, $e$ is the charge of electron, $n_{e}$ is the number density of electrons and $p_{e}$ is the electronic pressure. Since no applied or polarization voltage is imposed on the flow field, the electric field vector $\vec{E}=0$. For weakly ionized gases, the generalized Ohms law under the above conditions gives $J_{y}=0$ everywhere in the flow. Hence under these assumptions, equating the $x$ and $z$ components in (1) and solving for the current density components $J_{x}$ and $J_{z}$ readily read as, 
$J_{x}=\frac{\sigma B_{0}}{1+m^{2}}(m u-w)$

$J_{z}=\frac{\sigma B_{0}}{1+m^{2}}(u+m w)$

In above, $u, v$ and $w$ are the $x, y$ and $z$-components of the velocity vector $\vec{V}$ and $m=\omega_{e} \tau_{e}$ is the Hall parameter. Under these assumptions the governing boundary layer conservation of mass and momentum equations for both fluid and dust phase are written as ([9],[12]);

$\frac{\partial u}{\partial x}+\frac{\partial v}{\partial y}=0$

$u \frac{\partial u}{\partial x}+v \frac{\partial v}{\partial y}=v \frac{\partial^{2} v}{\partial y^{2}}+\frac{K N}{\rho}\left(u_{p}-u\right)-\frac{\sigma B_{0}}{1+m^{2}}(u+m w)$

$u \frac{\partial w}{\partial x}+v \frac{\partial w}{\partial y}=v \frac{\partial^{2} w}{\partial y^{2}}+\frac{K N}{\rho}\left(w_{p}-w\right)+\frac{\sigma B_{0}}{1+m^{2}}(m u-w)$

$\frac{\partial u_{p}}{\partial x}+\frac{\partial v_{p}}{\partial y}=0$

$u_{p} \frac{\partial u_{p}}{\partial x}+v_{p} \frac{\partial u_{p}}{\partial y}=\frac{K}{m_{p}}\left(u-u_{p}\right)$

$u_{p} \frac{\partial w_{p}}{\partial x}+v_{p} \frac{\partial w_{p}}{\partial y}=\frac{K}{m_{p}}\left(w-w_{p}\right)$

here, $(u, v, w)$ and $\left(u_{p}, v_{p}, w_{p}\right)$ are fluid and dust phase velocity components along $x-, y$ - and $z$-directions respectively. $v\left(=\frac{\mu}{\rho}\right)$ is the kinematic coefficient of viscosity, $\mu$ is the dynamic viscosity of the fluid, $\rho$ is the density of the fluid, $N$ is the number density of dust particle, $K=6 \pi \mu r$ is the Stokes drag constant, $r$ is the radius of dust particles, $\sigma$ is the electric conductivity and $m_{p}$ is the mass of the dust particles. The equations (4)-(6) and (7)-(9) are respectively, represents governing equations for fluid phase and dust particle phase. The first two terms in the right-hand side of the momentum equation (5) are, respectively, viscosity term and the term represents the force due to the relative motion between clean fluid and dust particles. The last term represents the Hall Effect term.

The appropriate boundary conditions suggested by the physics of the problem are: 
where $U_{w}(x)=b x$ is the stretching sheet velocity, $b$ is positive constant, $V_{w}(x)$ is the surface mass flux. It should be noted that positive values of $V_{w}$ indicate fluid blowing at the sheet surface while negative values of $V_{w}$ correspond to fluid suction at the wall.

To examine the flow regime, it is possible to reduce the partial differential equations (4)-(9) to ordinary differential equations by using the following similarity transformations:

$$
\begin{gathered}
u=b x f^{\prime}(\eta), \quad v=-\sqrt{b v} f(\eta), \quad w=b x h(\eta), \quad \eta=\sqrt{\frac{u_{w}}{v x}} y \\
u_{p}=b x F^{\prime}(\eta), \quad v_{p}=-\sqrt{b v} F(\eta), w_{p}=b x H(\eta)
\end{gathered}
$$

The equations (4) and (7) are identically satisfied and equations (5), (6), (8) and (9) are respectively reduced to following multidegree ordinary differential equations in terms of equation (11).

$$
f^{\prime \prime \prime}(\eta)+f^{\prime \prime} \eta f(\eta)-f^{\prime}(\eta)^{2}+l \beta_{v}\left(F^{\prime}(\eta)-f^{\prime}(\eta)\right)-\frac{M^{2}}{\left(1+m^{2}\right)}\left(f^{\prime}(\eta)+m h(\eta)\right)=0
$$

$h^{\prime \prime}(\eta)+h^{\prime}(\eta) f(\eta)-f^{\prime}(\eta) h(\eta)+l \beta_{v}(H(\eta)-h(\eta))+\frac{M^{2}}{\left(1+m^{2}\right)}\left(m f^{\prime}(\eta)-h(\eta)\right)=0$

$$
\begin{aligned}
& F^{\prime \prime}(\eta) F(\eta)-F^{\prime}(\eta)^{2}+\beta_{v}\left(f^{\prime}(\eta)-F^{\prime}(\eta)\right)=0 \\
& H^{\prime}(\eta) F(\eta)-H(\eta) F^{\prime}(\eta)+\beta_{v}(h(\eta)-H(\eta))=0
\end{aligned}
$$

with boundary conditions,

$$
\begin{aligned}
& f^{\prime}(\eta)=1, f(\eta)=S, h(\eta)=0, \text { at } \eta=0 \\
& f^{\prime}(\eta) \rightarrow 1, \quad F^{\prime}(\eta) \rightarrow 1, \quad F(\eta) \rightarrow f(\eta) \\
& h(\eta) \rightarrow 0, H(\eta) \rightarrow 0, \text { as } n=\infty
\end{aligned}
$$

where prime denotes the differentiation with respect to $\eta, l=\frac{m_{p} N}{\rho}$ is the mass concentration of the dust particle, $\tau_{v}=\frac{m_{p}}{K}$ relaxation time of dust particle, $\beta_{v}=\frac{1}{b \tau_{v}}$ is the fluid-particle interaction parameter 
for velocity, $M^{2}=\frac{B_{0}^{2} \sigma}{\rho b}$ is the magnetic parameter and $S=-\frac{v_{w}(x)}{\sqrt{b v}}$ is suction/blowing parameter.

If $m=0$ and $l=0$ in equation (12) with respect boundary condition (16), admits the closed-form analytical solution:

$f(\eta)=\frac{1-e^{\zeta \eta}}{\zeta}$

where $\zeta=\sqrt{\left(M^{2}+1\right)}$

Physical quantities of interest is the local skin friction coefficient in $x$-direction $C_{f_{x}}$ local skin friction coefficient in $z$-direction $C_{f_{z}}$ which is defined as

$C_{f_{x}}=\frac{\tau_{w_{x}}}{\rho U_{w^{2}}}, \quad C_{f_{z}}=\frac{\tau_{w_{z}}}{\rho U_{w^{2}}}$

where $\tau_{w_{x}}$ and $\tau_{w_{z}}$ are the surface shear stress in $x$ and $z$ directions respectively, which are given by

$\left.\tau_{w_{x}}=\mu \frac{\partial u}{\partial y}\right)_{y=0}, \quad \tau_{w_{z}}=\mu\left(\frac{\partial w}{\partial y}\right)_{y=0}$

Using the similarity variables, we obtain

$\sqrt{\operatorname{Re}_{x}} C_{f_{x}}=f^{\prime \prime}(0), \quad \sqrt{\operatorname{Re}_{x}} C_{f_{z}}=h^{\prime}(0)$

where $R e_{x}=\frac{u_{w}(x)}{v}$ is the local Reynolds number.

\section{Results and Discussion}

The set of non-linear differential equations (12)-(15) subject to the boundary conditions (16) are integrated numerically using a very efficient method known as Runge-Kutta-Fehlberg fourth-fifth order method. The asymptotic boundary conditions at $\eta \rightarrow \infty$ were replaced by those at $\eta=6$ in accordance with standard practice in the boundary layer analysis. Mainly five governing parameters are arise in this study namely, Hall parameter $(m)$, magnetic field parameter $\left(M^{2}\right)$, suction/blowing parameter $(S)$, mass concentration of dust particles parameter $(l)$ and fluid particle interaction parameter $\left(\beta_{v}\right)$. The influence of these parameters on the velocities of both fluid phase and dust phase are presented in Figs. 2-11. In addition Figs. 12 and 13 display the response of the local skin-friction coefficient along $x$ direction $f^{\prime \prime}(0)$ and the local skin-friction coefficient along $z$-direction 
$h^{\prime}(0)$ for different values physical parameters.
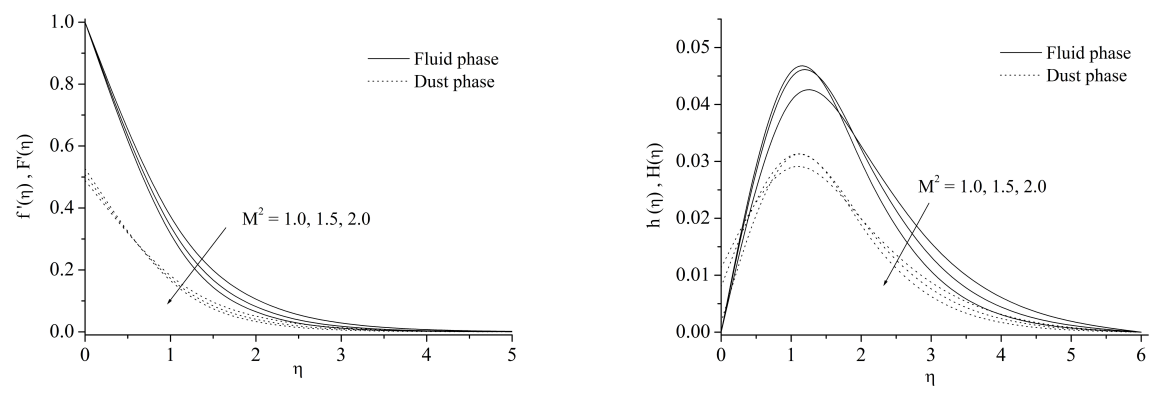

Figures 2 and 3: Variation of axial and transverse velocity profile for different values of $M^{2}$.

Figs. 2 and 3 shows the effect of the magnetic parameter on the axial and transverse velocity profiles for both fluid and dust phases. From the plot 2 , it is evident that increasing values of magnetic parameter results in flattening of tangential velocity of both phases. This is due to the influence of Lorentz force which is produced by means of applied magnetic field. In the absence of the magnetic field $\left(M^{2}=0\right)$ there is no transverse velocity for both phases $(h(\eta)=H(\eta)=0)$ and as the magnetic field increases (consequently $m$ increases), a cross flow in the transverse direction is greatly induced due to the Hall effect as depicted in the fig. 3. Additionally we can see that, increase in the values of $M^{2}$ leads to an increase in the values of the transverse velocity profiles for both phases nearer to the surface with shifting the maximum toward the sheet, however in the rest part of the sheet the transverse velocity profile decreases.
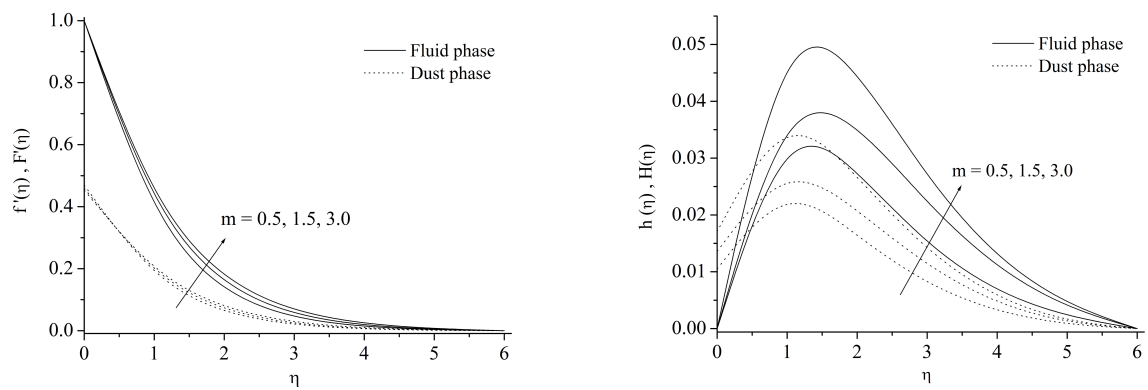

Figures 4 and 5: Variation of axial and transverse velocity profile for different values of $m$. 

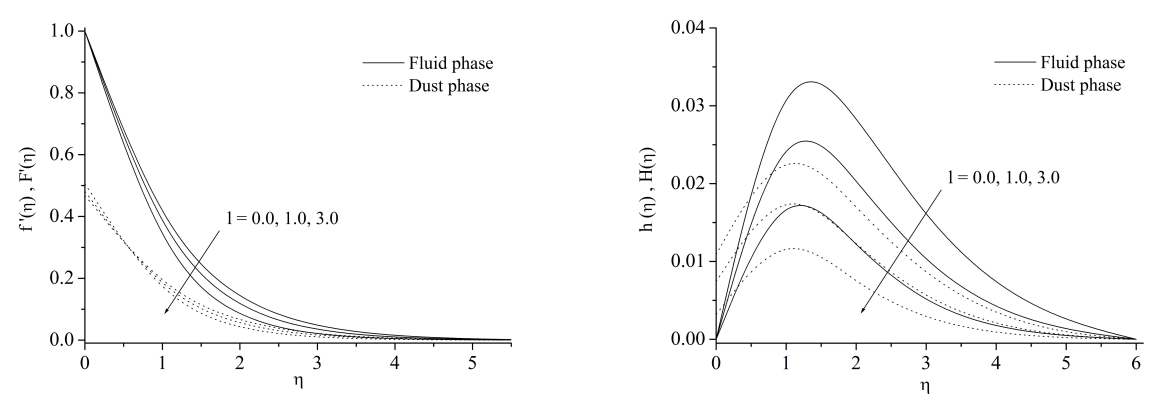

Figures 6 and 7: Variation of axial and transverse velocity profile for different values of $l$.

Figs. 4 and 5 are aimed to shed light on the effect of Hall parameter on axial and transverse velocities for both fluid phase and dust phases. It is seen that the axial velocity and transverse velocity profile for both fluid and dust phase increase with increasing values of Hall parameter. As the effective conductivity $\left(\frac{\rho}{\left(1+m^{2}\right)}\right)$ decreases with increasing $m$ which reduces the magnetic damping force on axial and transverse velocity profiles. Moreover, the $f^{\prime}(\eta)$ and $F^{\prime}(\eta)$ profiles approach their classical hydrodynamic values when the Hall parameter tends to $\infty$. Since the magnetic force terms approach zero value for very large values of Hall parameter.
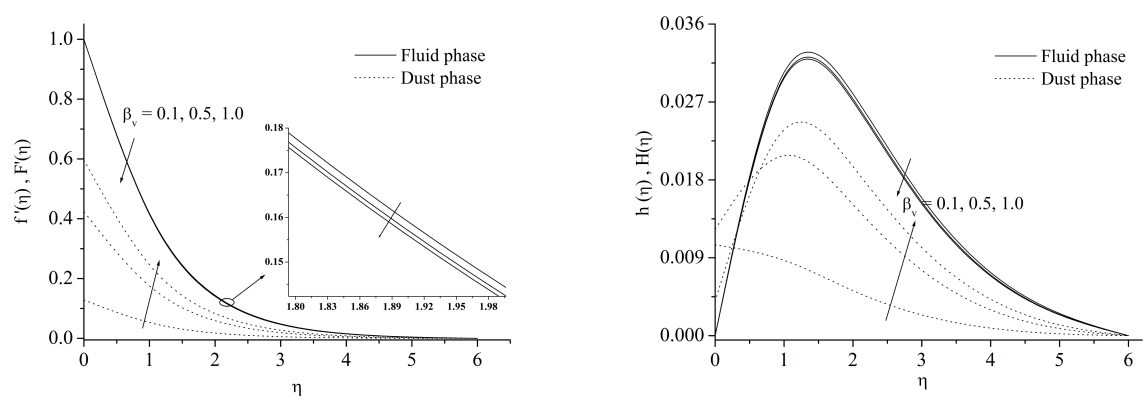

Figures 8 and 9: Variation of axial and transverse velocity profile for different values of $\beta_{v}$. 

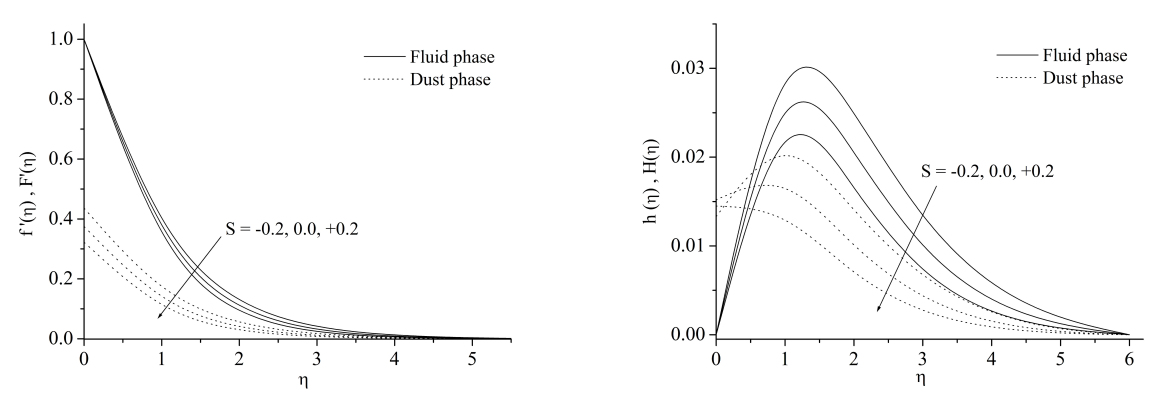

Figures 10 and 11: Variation of axial and transverse velocity profile for different values of $S$.

Figs. 6 and 7 illustrate the effect of mass concentration of dust particle parameter on the axial and transverse velocities for both fluid phase and dust phases. It is evident that increasing the values of $l$ results in decrease in the axial and transverse velocity profile for both the phases. In addition, the axial and transverse momentum boundary layer thickness decreases. This is due to fact that the drag force within the fluid is increases with increase in $l$. This drag force reduces the fluid phase velocities, which turn out reduction of dust phase velocities as well, since the dust phase velocity is dragged with fluid velocity. Further, it is observed that the axial and transverse velocity profile is higher for ordinary viscous fluid $(l=0)$ than that of dusty viscous fluid $(l \neq 0)$. The effect of fluid-particle interaction parameter on the axial and transverse velocity profiles are depicted in Figs. 8 and 9. It is observed that the fluid-particle interaction parameter contributes to thickening of axial and transverse momentum boundary layer for dust phase. However, this trend is quite opposite for fluid phase velocities.
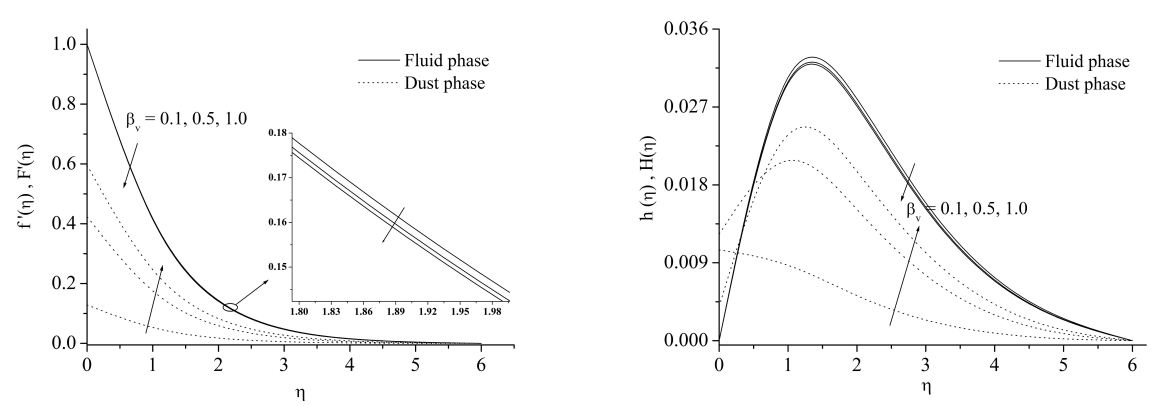

Figures 8 and 9: Variation of axial and transverse velocity profile for different values of $\beta_{v}$. 

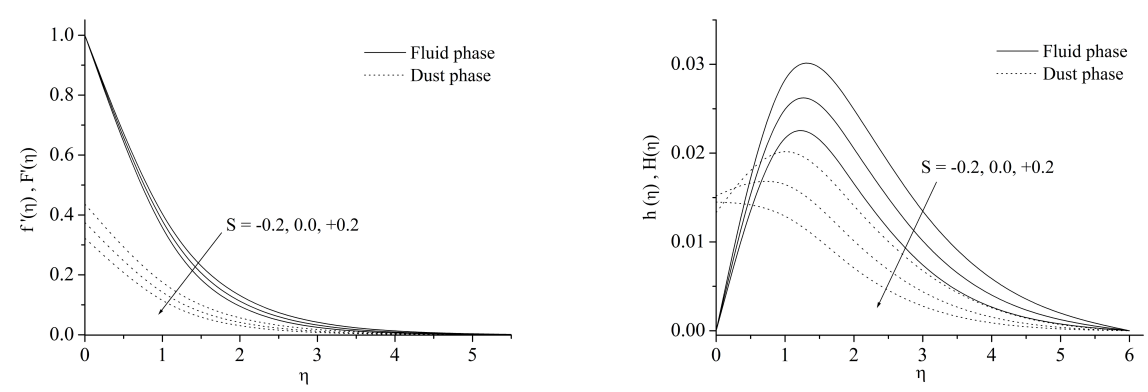

Figures 10 and 11: Variation of axial and transverse velocity profile for different values of $S$.

The influence of injection $(S<0)$ and suction $(S>0)$ on the axial and transverse velocity profiles is depicted in the Figs. 10 and 11 . As expected the opposite results are found for suction and blowing effect. The blowing effect causes an increase in axial and transverse velocity profile for both fluid and dust phase, but this trend is quite opposite for suction effect. This is due to the fact that the heated fluid is pushed farther away from the sheet when we provide stronger blowing effect. The same principle is acted but in reverse direction in the case of suction.

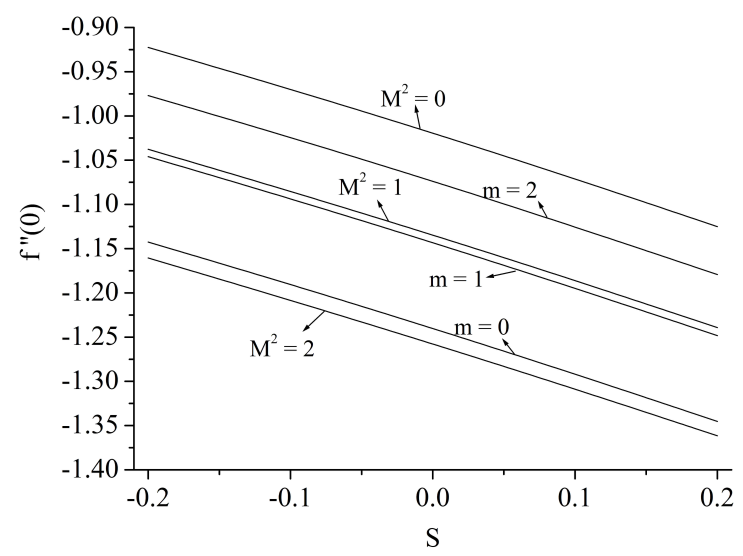

Figure 17: Variation of $f^{\prime \prime}(0)$ for different values of $M^{2}$ and $m$ vs $S$. 


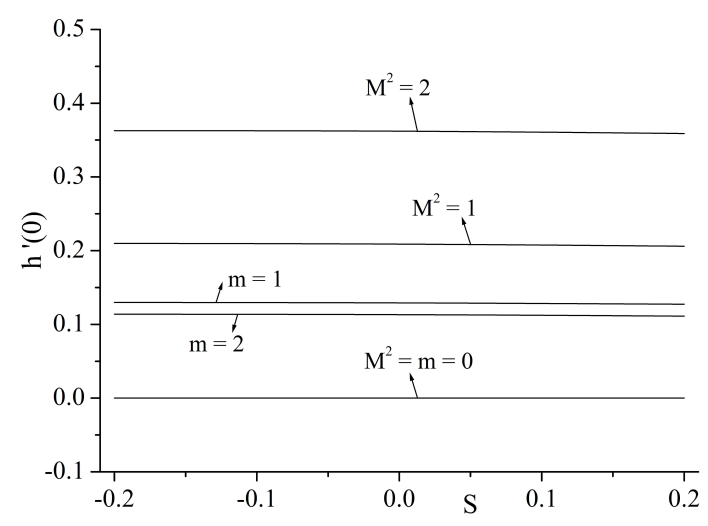

Figure 18: Variation of $h^{\prime}(0)$ for different values of $M^{2}$ and $m$ vs $S$.

Figs. 12 and 13, respectively, illustrate the influence of magnetic parameter and Hall parameter on local skin friction coefficient in the $x$ direction $f^{\prime \prime}(0)$ and the local skin friction coefficient in the $z$-direction $h^{\prime}(0)$ versus the suction/injection parameter. From the figure 12, one can see that the local skin friction coefficient along $x$-direction is greatly high in case of blowing and lower in case of the suction for all values of magnetic parameter and Hall parameter. Besides, from plot 12 , it is seen that, the skin friction coefficient is gradually increased for increasing values of Hall parameter. This is evident from the fact that increasing the values $\mathrm{m}$ would cause decreasing the resistive effect of the magnetic field. Furthermore, for increasing values magnetic parameter the local skin friction coefficient in the $x$-direction is decreases and $f^{\prime \prime}(0)$ profile is higher for in the absence of magnetic parameter, which is depicted in the fig. 12. We now move over to a discussion of the skin friction coefficient along the $z$-direction. It is seen from the graph 13 that, the transverse skin friction coefficient $h^{\prime}(0)$ is null in the absence of magnetic and Hall parameter. In addition, the $h^{\prime}(0)$ is lower in the case of suction and higher for blowing case. Further, It is seen from the plot that, $h^{\prime}(0)$ is increases for the increasing values of $M^{2}$ and decreases for increasing values of $m$.

\section{Conclusion}

A steady flow of an electrically conducting dusty viscous fluid over a permeable stretching sheet with Hall effect and suction/blowing has been investigated. In this study, the emphasis is given how fluid phase and dust phase velocity fields as well as the skin friction changes due to the influence of applied strong magnetic field along with Hall current and suction/blowing. The governing partial differential equa- 
tions are converted into ordinary differential equations by similarity transformation, before being solved numerically using the RungeKutta-Fehlberg fourth-fifth order method. As a summary, we can conclude that:

- The individual effects of increasing values of $M^{2}$ and $l$ is decreases the magnitude of both axial and transverse velocity profile in both phases. An opposite effect is observed for increasing values of $m$.

- For larger values of fluid-particle interaction parameter, the fluid phase axial and transverse velocity profile increases for dust phase and decreases for fluid phase.

-The effects of suction retards both fluid and dust phase velocity profile and blowing effect enhances the both fluid and dust phase velocity profile.

- The skin friction coefficient $f^{\prime \prime}(0)$ is gradually increases for increasing values of $\mathrm{m}$ and decreases for increasing values of $M^{2}$.

- Finally, momentum boundary layer is thinner due to the influence of suspended dust particles.

Thus the present study will serve as a good scientific tool for understanding more complex flow problems concerning with the various physical parameters.

\section{Acknowledgement}

I would like to express my sincere gratitude to Prof. B.J.Gireesha, Kuvempu University for giving me good guidelines to improve the quality of this paper. Also, I express my sincere thanks to the Management of the CHRIST UNIVERSITY for kind support.

\section{References}

[1] H. Sato, "The Hall effect in the viscous flow of ionized gas between two parallel plates under transverse magnetic field," J. Phyc. Sco. Jpn., vol. 16, no. 7, pp. 1427-1433, 1961.

[2] T. Yamanishi, "Hall effect in the viscous flow of ionized gas through straight channels," J. Physc. Sco. Jpn., vol. 16, no. 5, pp. 5-29, 1962.

[3] I. Pop and V. M. Soundalgekar, "Effects of Hall current on hydromagnetic flow near a porous plate," Act. Mech., vol. 20, no. 3, pp. 315318, 1974.

[4] A. K. Singh and R. S. R. Gorla, "Free convection heat and mass transfer with Hall current, joule heating and thermal diffusion," Heat Mass Transfer, vol. 45, no. 45, pp. 1341-1349, 2009.

[5] M. A. El-aziz, "Flow and heat transfer over an unsteady stretching surface with Hall effect," Meccanica., vol. 45, no. 1, pp. 97-109, 2010.

[6] F. M. Ali, R.Nazar, N. M. Arifin and I. Pop, "Effect of Hall current on MHD mixed convection boundary layer flow over a stretched vertical flat plate," Meccanica., vol. 46, no. 5, pp. 1103-1112, 2011. 
[7] E. M. Abo-Eldahab, and M. A. E. Aziz, "Hall current and Ohmic heating effects on mixed convection boundary layer flow of a micropolar fluid from a rotating cone with power-law variation in surface temperature," Heat Mass Transfer, vol. 31, no. 5, pp. 751-762, 2004.

[8] B. J. Gireesha and B. Mahanthesh, "Perturbation solution for radiating viscoelastic fluid flow and heat transfer with convective boundary condition in non-uniform channel with Hall current and chemical reaction," ISRN Thermodynamics., vol. 2013, no. 2013, pp. 14, 2013.

[9] P. G. Saffman, "On the stability of laminar flow of a dusty gas," J. Fluid Mech., vol. 13, no. 1, pp. 120-128, 1962.

[10] J. T. C. Liu, "Flow induced by an oscillating infinite flat plate in a dusty gas," Phys. Fluids., vol. 9, no. 9, pp. 1716-1720, 1966.

[11] K. M. Chakrabarti, "Note on boundary layer in a dusty gas," AIAA Journal, vol. 12, no. 8, pp. 1136-1137, 1974.

[12] N. Datta and S. K. Mishra, "Boundary layer flow of a dusty fluid over a semiinfinite flat plate," Acta Mechanica., vol. 42, no. 1, pp. 71-83, 1982.

[13] K. Vajravelu and J. Nayfeh, "Hydromagnetic flow of a dusty fluid over a stretching sheet," Int. J. Nonlin. Mech., vol. 27, no. 6, pp. 937-945, 1992.

[14] M. Xie,J. Lin, and F. Xing, "On the hydrodynamic stability of a particle-laden flow in growing flat plate boundary layer," J. Zhejiang Univ. - Sci. A, vol. 8, no. 2, pp. 275-284, 2007.

[15] G. Palani and P. Ganesan, "Heat transfer effects on dusty gas flow past a semiinfinite inclined plate," ForschIngenieurwes, vol. 71, no. 3, pp. 223-230, 2007.

[16] B. J. Gireesha, B. Mahanthesh, Rama Subba Reddy Gorla, and K. L. Krupalakshmi, "Mixed convection two-phase flow of Maxwell fluid under the influence of non-linear thermal radiation, non-uniform heat source/sink and fluidparticle suspension," Ain Shams Engineering Journal, to be published.

[17] N. Sandeep, C. Sulochana and B. Rushi Kumar, "Unsteady MHD radiative flow and heat transfer of a dusty nanofluid over an exponentially stretching surface" Eng. Sci. Tech., vol. 19, no. 1, pp. 227-240, 2016.

[18] S. Siddiqa, N. Begum, M. A. Hossain and R. S. R. Gorla, "Numerical solutions of natural convection flow of a dusty nanofluid about a vertical wavy truncated cone," J. Heat Transfer, vol. 139, no. 2, 2017.

[19] M. A. Hossain, N. C. Roy and S. Siddiqa, "Unsteady mixed convection dusty fluid flow past a vertical wedge due to small fluctuation in free stream and surface temperature," Appl. Math. Computation, vol. 293, pp. 480-492, 2017.

[20] T. Mustafa, "Magnetohydrodynamic two-phase dusty fluid flow and heat model over deforming isothermal surfaces," Physics of Fluids, vol. 29 no. 1, 2017. 